\title{
Thermal spectrum of pseudo-scalar glueballs and Debye screening mass from holography
}

\author{
Nelson R. F. Braga ${ }^{a}$, Luiz F. Ferreira ${ }^{b}$ \\ Instituto de Física, Universidade Federal do Rio de Janeiro, Caixa Postal 68528, Rio de Janeiro, RJ 21941-972, Brazil
}

Received: 13 June 2017 / Accepted: 15 September 2017 / Published online: 4 October 2017

(C) The Author(s) 2017. This article is an open access publication

\begin{abstract}
The finite temperature spectrum of pseudo-scalar glueballs in a plasma is studied using a holographic model. The $0^{-+}$glueball is represented by a pseudo-scalar (axion) field living in a five dimensional geometry that comes from a solution of Einstein equations for gravity coupled with a dilaton scalar field. The spectral function obtained from the model shows a clear peak corresponding to the quasi-particle ground state. Analyzing the variation of the position of the peak with temperature, we describe the thermal behavior of the Debye screening mass of the plasma. As a check of consistency, the zero temperature limit of the model is also investigated. The glueball masses obtained are consistent with previous lattice results.
\end{abstract}

\section{Introduction}

The AdS/CFT correspondence [1-4] inspired the development of holographic models that describe strong interaction properties based on gauge/string duality. Some of the first work in this direction assumed the existence of an approximate duality between a field theory living in some ad hoc deformation of anti-de Sitter (AdS) space containing a dimension-full parameter and a gauge theory where the parameter plays the role of an energy scale. The simplest example is the hard wall AdS/QCD model, which appeared in Refs. [5-7]. It consists in placing a hard geometrical cutoff in AdS space. This model provides, in a very simple way, glueballs masses consistent with lattice results. Another AdS/QCD model, the soft wall, where the square of the mass grow linearly with the radial excitation number, was introduced in Ref. [8]. In this case, the background involves AdS space and a scalar field that acts effectively as a smooth infrared cutoff. One finds an interesting review of AdS/QCD models and a wide list of related references in [9].

\footnotetext{
a e-mail: braga@if.ufrj.br

be-mail: luizfaulhaber@if.ufrj.br
}

A finite temperature version of the AdS/CFT correspondence was found in $[4,10]$. In this case the gauge theory dual is a black-hole geometry. The corresponding finite temperature versions of AdS/QCD models provide a nice picture of the confinement/deconfinement thermal phase transition [11-13].

The holographic approach to the strong interaction has been widely improved over the years. In particular, models like [14-20] that use supergravity backgrounds coming from consistent solutions of the Einstein equations, mimic very important results of QCD, like the running of the coupling constant. These type of improved models require in general numerical solutions for the supergravity background.

Interesting analytical and numerical results for the thermal behavior of the plasma were obtained also in [21-27]. For example, in Ref. [25] the spectral function of the shear operator $T_{12}$ was calculated in a hot Yang-Mills theory.

In Ref. [28] the thermodynamics of the plasma was studied using a simplified model that has a first order phase transition. The results obtained for the sound speed, the entropy and other thermodynamic quantities are in good agreement with lattice results [29], showing that the model captures in a consistent way some important plasma properties.

The purpose of the present article is to apply the model of Ref. [28] to another important property of the plasma, namely, the Debye screening mass. The approach that we will follow is to study the thermal spectrum of pseudo-scalar $0^{-+}$glueballs. These particles are dual to the axion field in gauge gravity duality. The mass of the ground state of $0^{-+}$glueballs corresponds to the Debye screening mass of the plasma [30,31]. We will follow the prescriptions used in Refs. [14, 15,32,33] for the action of an axion field. The spectral function obtained presents a peak corresponding to the ground state of the pseudo-scalar glueball. So, the variation of the position of the peak with the temperature shows the thermal behavior of the Debye screening mass. 
The article is organized as follows. In Sect. 2 we review the model of reference [28]. Then in Sect. 3 we describe an axion field in this model and obtain the thermal spectrum of pseudoscalar glueballs. In Sect. 4 we study the zero temperature limit as a check of consistency of the model. Then we discuss the results obtained in the article in Sect. 5 and analyze the temperature dependence of the Debye screening mass.

\section{Holographic model with thermal phase transition}

The holographic bottom up model presented in Ref. [28] is constructed using a 5-dimensional Einstein plus dilaton effective bulk action. The model is a simplified version of the improved holographic QCD (IHQCD) models of Refs. [14, 15] that presents analytical solutions for the background.

The effective five dimensional action for the metric and the dilaton in the Einstein frame is

$S=\frac{1}{16 \pi G_{5}} \int \mathrm{d}^{5} x \sqrt{-g}\left[R-\frac{4}{3}(\partial \phi)^{2}-V_{E}(\phi)\right]$,

where $G_{5}$ is the five dimensional Newton's constant and $\phi$ the dilation field. The metric is assumed to have the form

$\mathrm{d} s_{E}^{2}=\frac{e^{2 A_{E}(z)}}{z^{2}}\left[-f(z) \mathrm{d} t^{2}+\mathrm{d} \vec{x}^{2}+\frac{\mathrm{d} z^{2}}{f(z)}\right]$,

and the boundary is located at $z=0$.

The potential $V_{E}(\phi)$ in the action (1) is not chosen a priori. The equations of motion relate $A_{\mathrm{E}}(z), \phi(z), f(z)$ and $V_{E}(\phi)$. The strategy to be followed is to choose a specific form for the warp factor and then determine the other quantities. It is convenient to replace the Einstein frame warp factor $A_{\mathrm{E}}$ by the corresponding factor in the string frame $A_{s}$ :

$A_{\mathrm{E}}(z)=A_{s}(z)-\frac{2}{3} \phi(z)$.

The equations of motion for the Einstein frame action (1) are

$E_{\mu \nu}+\frac{1}{2} g_{\mu \nu}\left(\frac{4}{3} \partial_{\mu} \phi \partial^{\mu} \phi+V_{E}(\phi)\right)-\frac{4}{3} \partial_{\mu} \phi \partial_{\nu} \phi=0$,

$\frac{8}{3} \partial_{z}\left(\frac{e^{3 A_{s}-2 \phi} f}{z^{3}} \partial_{z} \phi\right)-\frac{e^{5 A_{s}-\frac{10}{3} \phi}}{z^{5}} \partial_{\phi} V_{E}=0$,

where the Einstein tensor is $E_{\mu \nu}=R_{\mu \nu}-\frac{1}{2} g_{\mu \nu} R$ and the prime indicates differentiation with respect to $z$.

The non-zero components of the gravity equation of motion (4) read

$$
\begin{aligned}
& A_{s}^{\prime \prime}+A_{s}^{\prime}\left(\frac{f^{\prime}}{2 f}-\frac{2}{z}+A_{s}^{\prime}-\frac{4}{3} \phi^{\prime}\right)-f^{\prime}\left(\frac{\phi^{\prime}}{3 f}+\frac{1}{2 z f}\right) \\
& -\frac{2 \phi^{\prime \prime}}{3}+\frac{2}{3} \phi^{\prime}\left(\phi^{\prime}+\frac{2}{z}\right) \\
& +\frac{2}{z^{2}}+\frac{V_{E}(\phi)}{6 z^{2} f} e^{2 A_{s}-\frac{4}{3} \phi}=0, \\
& \phi^{\prime 2}-\phi^{\prime}\left(4 A_{s}^{\prime}+\frac{f^{\prime}}{2 f}-\frac{4}{z}\right)+A_{s}^{\prime}\left(\frac{3 f^{\prime}}{4 f}-\frac{6}{z}+3 A_{s}^{\prime}\right) \\
& -\frac{3 f^{\prime}}{4 z f}+\frac{3}{z^{2}}+\frac{V_{E}(\phi)}{4 z^{2} f} e^{2 A s-\frac{4}{3} \phi}=0, \\
& f^{\prime \prime}+f^{\prime}\left(6 A_{s}^{\prime 2}-\frac{6}{z}-4 \phi^{\prime}\right)+e^{2 A_{s}-\frac{4}{3} \phi} \frac{V_{E}(\phi)}{z^{2}} \\
& +f\left(6 A_{s}^{\prime \prime}+6 A_{s}^{\prime 2}+\frac{12}{z^{2}}-4 \phi^{\prime \prime}+4 \phi^{\prime 2}\right. \\
& \left.+\frac{8 \phi^{\prime}}{z}-\frac{4 A_{s}^{\prime}\left(2 z \phi^{\prime}+3\right)}{z}\right)=0 .
\end{aligned}
$$

Note that we only need two of the above three equations. The other equation can be used as a consistency check for the solutions. We recombine Eqs. (6), (7) and (8) and find the following simplified equations:

$$
\begin{aligned}
& f^{\prime \prime}+f^{\prime}\left(3 A_{s}^{\prime}-2 \phi^{\prime}-\frac{3}{z}\right)=0, \\
& \phi^{\prime \prime}-\phi^{\prime}\left(2 A_{s}^{\prime}-\frac{2}{z}\right)-\frac{3 A_{s}^{\prime \prime}}{2}-\frac{3 A_{s}^{\prime}}{z}+\frac{3}{2} A_{s}^{2}=0 .
\end{aligned}
$$

These equations determine the geometry once $A_{s}(z)$ is fixed. Integrating the previous equations one finds the solution in terms of $A_{s}(z)$ :

$$
\begin{aligned}
\phi(z)= & \phi_{0}+\frac{3 A_{s}(z)}{2}+\phi_{1} \int_{0}^{z} \frac{e^{2 A_{s}(x)}}{x^{2}} \mathrm{~d} x \\
& +\frac{3}{2} \int_{0}^{z} \frac{e^{2 A_{s}(x)} \int_{0}^{x} y^{2} e^{-2 A_{s}(y)} A_{s}^{\prime 2}(y) \mathrm{d} y}{x^{2}} \mathrm{~d} x, \\
f(z)= & f_{0}+f_{1}\left(\int_{0}^{z} x^{3} e^{-3 A_{s}(x)+2 \phi(x)} \mathrm{d} x\right),
\end{aligned}
$$

where $\phi_{0}, \phi_{1}, f_{0}, f_{1}$ are constants of integration. Note that $\phi(z)$ in the second equation is obtained from the first equation, as a function of $A_{s}(z)$. The potential $V_{E}(\phi)$ is also fixed by a choice of $A_{s}$. One just need to use the solutions (11) and (12) in the equation

$$
\begin{aligned}
V_{E}(\phi)= & e^{\frac{4}{3} \phi(z)-2 A_{s}(z)}\left(z^{2} f^{\prime \prime}(z)-4 f(z)\left(3 z^{2} A_{s}^{\prime \prime}(z)\right.\right. \\
& \left.\left.-2 z^{2} \phi^{\prime \prime}(z)+z^{2} \phi^{\prime 2}(z)+3\right)\right),
\end{aligned}
$$

The choice used in Ref. [28] for the warp factor $A_{s}$ is

$$
A_{s}(z)=k^{2} z^{2}
$$

Imposing the asymptotic $\mathrm{AdS}_{5}$ condition $f(0)=1$ near the $U V$ boundary $z \sim 0$ and requiring $\phi$ and $f$ to be finite at $z=0$ one finds 

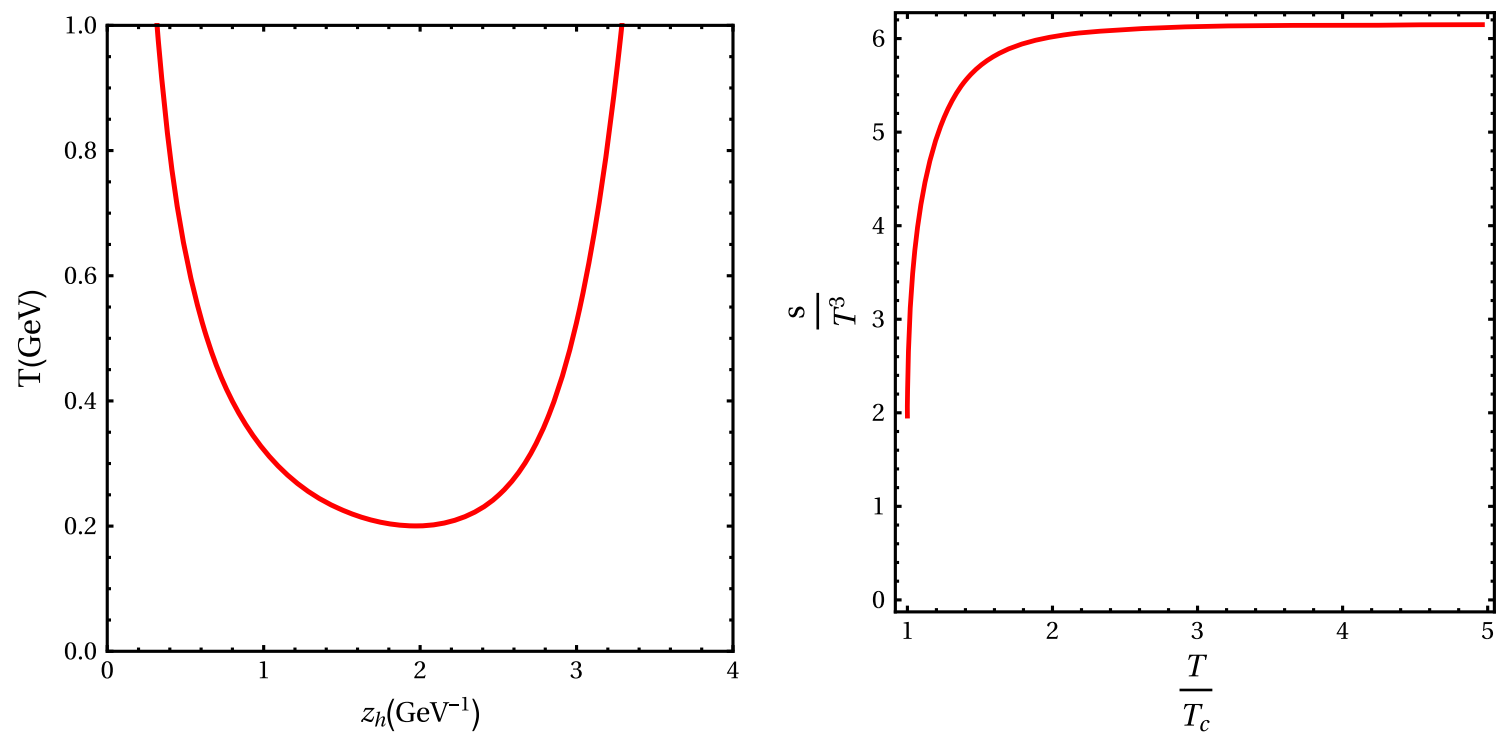

Fig. 1 The left panel shows the temperature $T$ as a function of the black-hole horizon $z_{h}$ with $k=0.43 \mathrm{GeV}$. In the right panel we present the scaled entropy density $s / T^{3}$ as a function of scaled temperature $T / T_{c}$ with $k=0.43 \mathrm{GeV}$ and $G_{5} / L^{3}=1.26$

$f(z)=1-\frac{\int_{0}^{k z} x^{3} \exp \left(\frac{3}{2} x^{2}\left(H_{c}(x / k)-1\right)\right)}{\int_{0}^{k z h} x^{3} \exp \left(\frac{3}{2} x^{2}\left(H_{c}(x / k)-1\right)\right)}$,

$\phi(z)=\frac{3}{4} k^{2} z^{2}\left(1+H_{c}(z)\right)$,

where

$H_{c}(z)={ }_{2} F_{2}\left(1,1 ; 2, \frac{5}{2} ; k^{2} z^{2}\right)$.

The solution for the dilaton potential takes the form

$$
\begin{aligned}
V_{E}(z)= & \frac{3 e^{k^{2} z^{2}\left(1-H_{c}(z)\right)}}{128 k^{2} z^{2}} f(z)\left[40 k^{2} z^{2}+64 k^{4} z^{4}\right. \\
& -384 k^{6} z^{6}-27 \pi e^{4 k^{2} z^{2}} \operatorname{Er} f_{c}(\sqrt{2} k z)^{2} \\
& \left.+12 \sqrt{12} e^{2 k^{2} z^{2}} k z\left(-7+20 k^{2} z^{2}\right) \operatorname{Er} f_{c}(\sqrt{2} k z)\right] \\
& -\frac{3 f^{h} e^{\frac{5}{2} k^{2} z^{2}\left(-1+H_{c}(z)\right)} k^{3} z^{3}}{16} \\
& \times\left[4 k z-16 k^{3} z^{3}+3 \sqrt{2 \pi} e^{2 k^{2} z^{2}} \operatorname{Er} f_{c}(\sqrt{2} k z)\right],
\end{aligned}
$$

where $\operatorname{Er} f_{c}[z]$ is error function which is defined as a integral form $\operatorname{Er} f[z]=\frac{2}{\sqrt{\pi}} \int_{0}^{z} e^{-t^{2}} \mathrm{~d} t, f^{h}$ is

$f^{h}=\frac{1}{\int_{0}^{k z_{h}} x^{3} \exp \left(\frac{3}{2} x^{2}\left(H_{c}(x / k)-1\right)\right)}$.

The temperature is obtained from

$T=\frac{\left|f^{\prime}\left(z_{h}\right)\right|}{4 \pi}$.
Using Eq. (15), one can find the relation between the temperature and the position of the black-hole horizon in this model,

$T\left(z_{h}\right)=\frac{k^{4} z_{h}^{3} \exp \left(\frac{3}{2}\left(k^{2} z_{h}^{2} H_{c}\left(z_{h}\right)-k^{2} z_{h}^{2}\right)\right)}{4 \pi \int_{0}^{k z_{h}} x^{3} \exp \left(\frac{3}{2} x^{2}\left(H_{c}(x / k)-1\right)\right)}$.

The entropy is given by

$s=\left.\frac{A_{\text {area }}}{4 G_{5} V_{3}}\right|_{z h}=\left.\frac{L^{3}}{4 G_{5}}\left(\frac{e^{A_{E}(z)}}{z}\right)^{3}\right|_{z_{h}}$.

Using $k=0.43 \mathrm{GeV}$ and $G_{5} / L^{3}=1.26$ as in Ref. [28] one obtains the numerical results for the temperature and entropy shown in Fig. 1.

Note from Fig. 1 that there is a minimal temperature $T_{\min }$ at a certain black-hole horizon position $z_{h}^{m}$. For $T<T_{\min }$, there are no black-hole solutions while for $T>T_{\min }$, there are two black-hole solutions. When $z_{h}<z_{h}^{m}$, the temperature increase with the decrease of $z_{h}$, this phase is thermodynamically stable. When $z_{h}>z_{h}^{m}$, the temperature increases with the increase of $z_{h}$; this phase is thermodynamically unstable and thus not physical.

The pressure density $p(T)$ can be calculated from the entropy density $s(T)$ by solving the equation

$\frac{\mathrm{d} p(T)}{\mathrm{d} T}=-s(T)$

After integrating Eq. (23), the pressure density of the system can be obtained up to an integral constant $p_{0}$. One can set $p_{0}=0$ to ensure that $p\left(T_{\min }\right)=0$. Thus, the critical 


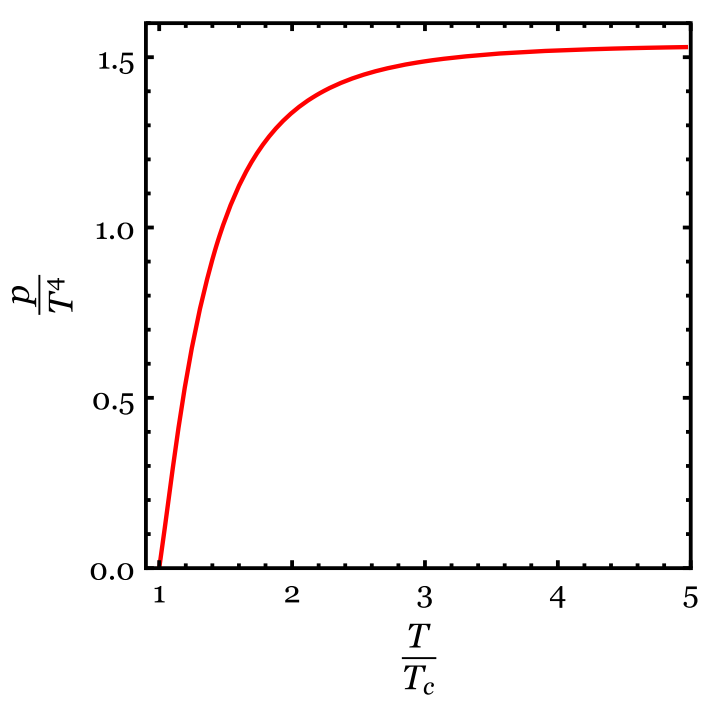

Fig. 2 The scaled pressure density $p / T^{4}$ as a function of scaled temperature $T / T_{c}$ with $k=0.43 \mathrm{GeV}$ and $G_{5} / L^{3}=1.26$

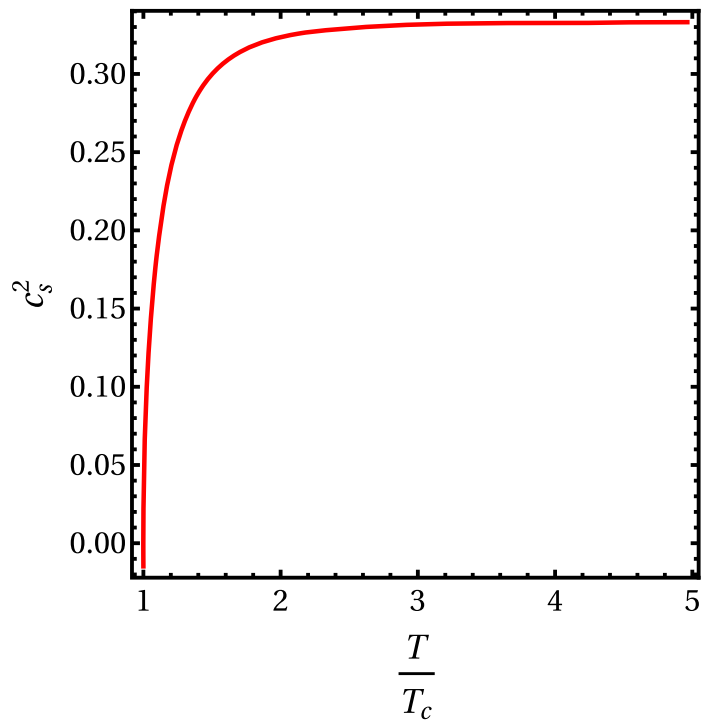

Fig. 3 The square of the sound velocity $c_{s}^{2}$ as a function of scaled temperature $T / T_{c}$ with $k=0.43 \mathrm{GeV}$ and $G_{5} / L^{3}=1.26$

temperature of this model is in $T_{c}=T_{\min }=201 \mathrm{MeV}$. In Fig. 2, we present the numerical result of pressure density as a function of temperature.

The sound velocity $c_{s}$ can be derived from the temperature and entropy:

$c_{s}^{2}=\frac{\mathrm{d} \log T}{\mathrm{~d} \log s}$.

From Eq. (24) one can see that the sound velocity is independent of the $5 D$ Newton constant $G_{5}$. In Fig. 3, we show the equation of state for this model.

\section{Debye mass from pseudo-scalar glueballs}

\subsection{Debye screening mass}

An important concept in the description of the deconfined phase of non-Abelian gauge theories at finite temperature is the Debye screening mass $m_{\mathrm{D}}$. The inverse of this quantity can be used to define a screening length felt by color-electric excitations, in analogy with the Debye screening in Abelian plasma, which is felt by electric fields but not by magnetic fields.

A gauge invariant non-perturbative definition for $m_{\mathrm{D}}$ was given in Ref. [30] where this quantity is defined as the smallest inverse correlation length in symmetry channels which are odd under Euclidean time reflection.

In this work we follow [31] and identify the smallest thermal mass, associated with the Pontryagin density operator $\operatorname{Tr}\left(F_{\mu \nu} \tilde{F}^{\mu \nu}\right)$, as the Debye mass in a strongly coupled plasma. Thus, we can use the spectral function of the pseudo-scalar glueball to find the Debye screening mass of the plasma. The supergravity field associated with the $0^{-+}$ operator is the axion.

\subsection{Spectral function for pseudo-scalar glueballs}

In order to describe the $0^{-+}$glueball we consider the axion field in the supergravity background presented in the previous section. The action for the massless axion fluctuation, $a$, is assumed to be of the same form as in Improved Holographic QCD [14-18]

$S=\frac{1}{32 \pi G_{5}} \int \mathrm{d}^{5} x \sqrt{g} \mathcal{Z}(z) g^{\mu \nu} \partial_{\mu} a \partial_{\nu} a$,

where the axion coupling $\mathcal{Z}$ is a function which represents a partial resummation of high orders forms coming from string theory $[14,15]$. The convenient parametrization for the axion coupling found in these references is

$\mathcal{Z}=1+c \lambda^{4}$

where $c$ is a constant and $\lambda=\lambda(z)=e^{\phi(z)}$ is the 't Hooft coupling.

The equation of motion that come from action (25) with metric (2) is

$\partial_{\mu}\left(\mathcal{Z} b^{5} g^{\mu \nu} \partial_{\nu} a\right)=0$

where $b(z)=e^{A_{E}(z)} / z$. In Fourier space Eq. (27) reads

$\partial_{z}\left(\mathcal{Z} b^{3} f \partial_{z} a\right)+\left(\frac{\omega^{2}}{f}-\vec{k}^{2}\right) \mathcal{Z} b^{3} a=0$. 
In order to obtain the spectral function of the pseudo-scalar glueball we follow the procedure of the membrane paradigm [34] as explained in Ref. [35]. We first introduce the bulk response function

$\xi(z, \omega, \vec{k}) \equiv \frac{\Pi(z, \omega, \vec{k})}{\omega a(z, \omega, \vec{k})}$,

where $\Pi(z, \omega, \vec{k})$ is the radial canonical momentum conjugate to the axion fluctuation $a(z, \omega, \vec{k})$ :

$\Pi(z, \omega, \vec{k}) \equiv \frac{\delta S}{\delta\left(\partial_{z} a\right)}=-\mathcal{Z} b^{3} f \partial_{z} a(z, \omega, \vec{k})$.

This procedure allows us to reduce the linear second order differential equation (28) to a first order nonlinear equation:

$\partial_{z} \xi-\frac{\omega}{\mathcal{Z} b^{3} f}\left[\xi^{2}+\mathcal{Z}^{2} b^{6}\left(1-f \frac{\vec{k}^{2}}{\omega^{2}}\right)\right]=0$.

Requiring regularity at the horizon, one obtains the following horizon condition, needed to solve the first order equation above:

$\xi\left(z_{h}\right)=i\left[\mathcal{Z} b^{3}\right]_{z=z_{h}}$

From the Kubo formula for the retarded Green function we have the following relation:

$G_{R}(\omega, \vec{k})=-\lim _{z \rightarrow 0} \frac{\Pi(z, \omega, \vec{k})}{a(z, \omega, \vec{k})}=-\omega \lim _{z \rightarrow 0} \xi(z, \omega, \vec{k})$.

Thus, the spectral function is obtained solving Eq. (31) and using the imaginary part of the Green function (33):

$\rho(\omega)=-2 \operatorname{Im} G_{R}(\omega, \vec{k}=0)$.

\subsection{Results}

We fix the value of the parameter in Eq. (26) as $c=0.26$ in order to find a zero temperature limit for the mass of the pseudo-scalar glueball consistent with the lattice results, as discussed in Refs. [14,15]. We also use $k=0.43 \mathrm{GeV}$.

The next step is to solve numerically Eq. (31) using the metric (2) and the axion coupling (26) to find the spectral function (34). We show in Fig. 4 the spectral function for the case $T=T_{c}=201 \mathrm{MeV}$. Note that we divide the spectral function by $T_{c}^{4}$ to have a dimensionless quantity. The numerical results show that for large $\omega$ the spectral function scales as $\omega^{4}$. So, we define a re-scaled spectral function, in a similar way as done is Refs. [36,37]:

$\tilde{\rho}(\omega)=\frac{\rho(\omega)}{\omega^{4}}$.

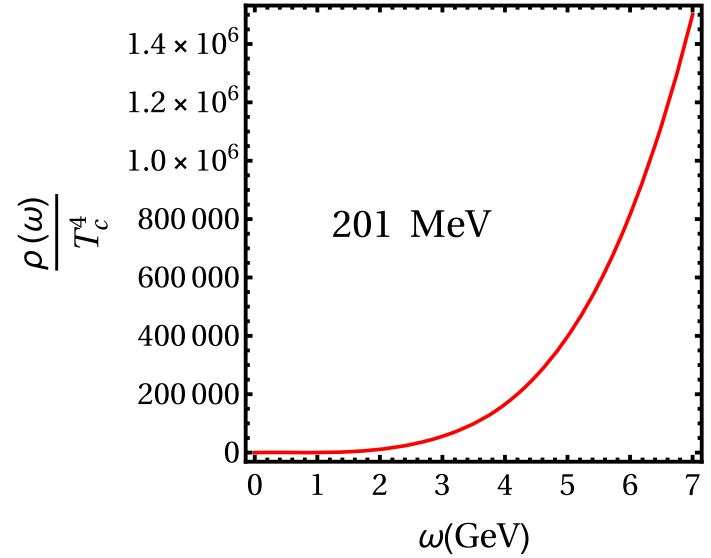

Fig. 4 Spectral function divided by $T_{c}^{4}$ as a function of the energy for the critical temperature

The results obtained for $\tilde{\rho}$ from numerical calculations at different temperatures are shown in Fig. 5. The location of the peak corresponds to the thermal mass of the ground state. Increasing the temperature of the plasma one can observe in Fig. 5 that the peak decreases and virtually disappears for temperatures greater than $T=230 \mathrm{MeV}$.

\section{Pseudo-scalar glueball masses at $T=0$}

As a check of the method used for describing pseudo-scalar glueballs inside a plasma, let us consider the limit of zero temperature. The equation of motion for the axion is (28)

$\partial_{z}\left(\mathcal{Z} b^{3} f \partial_{z} a\right)+\left(\frac{\omega^{2}}{f}-\vec{k}^{2}\right) \mathcal{Z} b^{3} a=0$.

In order to calculate the glueball $0^{-+}$masses at $T=0$ one takes $f(z)=1$ and Eq. (36) becomes

$\partial_{z}\left(\mathcal{Z} b^{3} \partial_{z} a\right)+\left(\omega^{2}-\vec{k}^{2}\right) \mathcal{Z} b^{3} a=0$.

Defining $\psi=e^{B} a$ and

$B(z)=\frac{3}{2} \log b(z)+\frac{1}{2} \log \mathcal{Z}(z)$

the equation of motion (37) takes the form

$-\psi^{\prime \prime}(z)+\mathcal{V}(z)=M^{2} \psi$

where $M^{2}=\omega^{2}-\vec{k}^{2}$ and the potential is defined as

$\mathcal{V}(z)=B^{\prime 2}+B^{\prime \prime}$ 

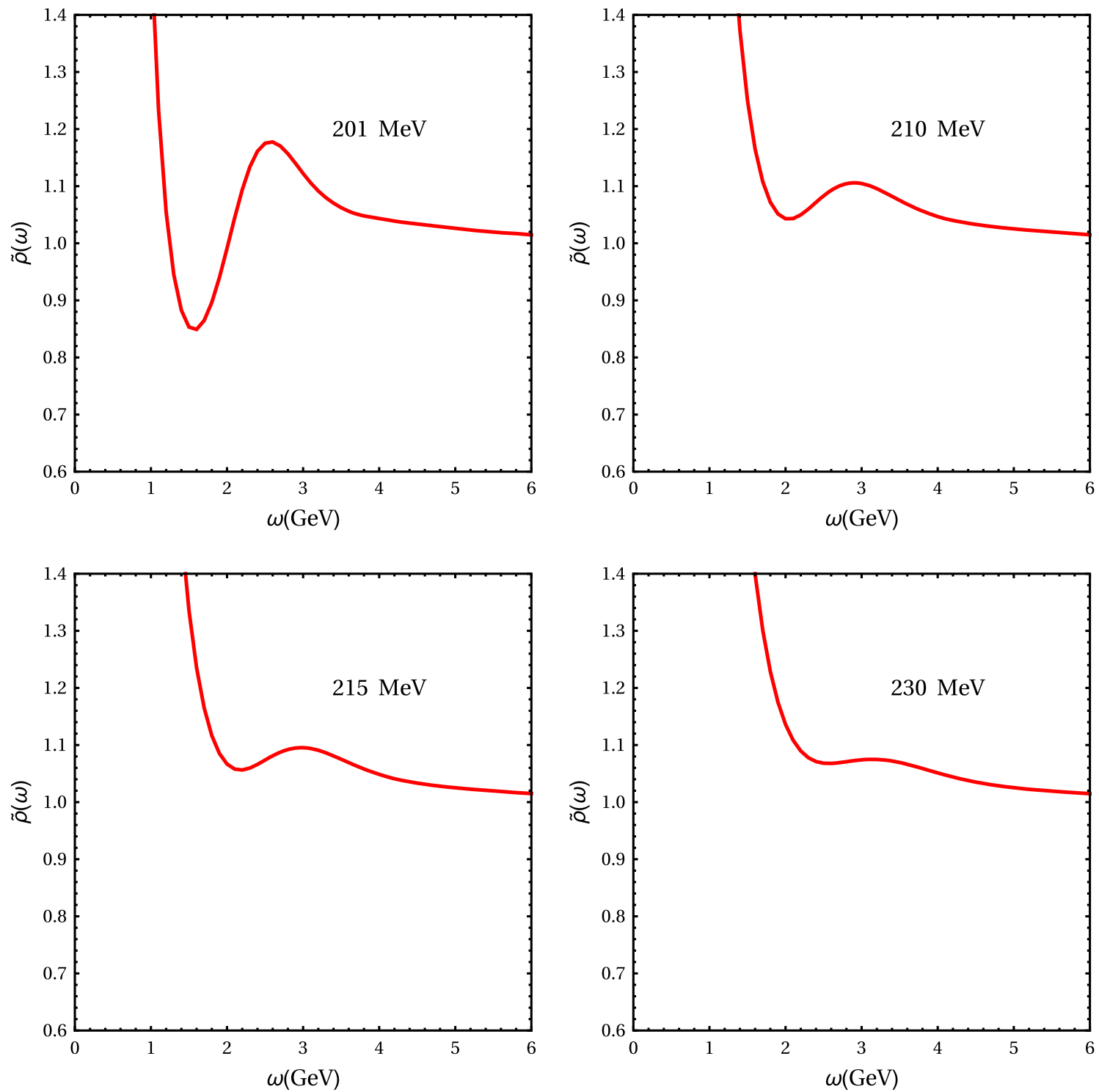

Fig. 5 Rescaled spectral function as a function of the energy $\omega$ in $\mathrm{GeV}$

Now, we can calculate the glueball mass using Eq. (39) and the metric (2) with $f(z)=1$. The form of $A_{\mathrm{E}}$ and $\phi$ is the same as in the finite temperature case.

The parametrization for the axion coupling $\mathcal{Z}(\lambda(z))$ is the same as in Eq. (26) with the same choice $c=0.26$ as in the finite temperature case of the previous section. We present the results for the masses of $0^{-+}$glueballs in Table 1, compared with lattice results $[38,39]$. One notes that there is a reasonable agreement.

\section{Analysis of the results}

The location of the peak of the spectral functions obtained in section III corresponds to the thermal mass of the ground
Table 1 Our holographic results for Glueball masses at zero temperature, with $k=0.43 \mathrm{GeV}$, compared with lattice data $[38,39]$

\begin{tabular}{llll}
\hline$J^{P C}$ & $\begin{array}{l}\text { Holographic } \\
\text { mass }(\mathrm{GeV})\end{array}$ & $\begin{array}{l}\text { Lattice mass } \\
{[38](\mathrm{GeV})}\end{array}$ & $\begin{array}{l}\text { Lattice mass } \\
{[39](\mathrm{GeV})}\end{array}$ \\
\hline $0^{-+}$ & 2.477 & 2.590 & 2.560 \\
$0^{*-+}$ & 3.617 & 3.640 & \\
$0^{* *-+}$ & 4.630 & & \\
\hline
\end{tabular}

state of the pseudo-scalar glueball, associated with the Debye screening mass of the plasma.

In order to exhibit the thermal behavior of the Debye mass we plot in Fig. 6 some values of the peak locations at different temperatures. The smallest value of the mass occurs at the 


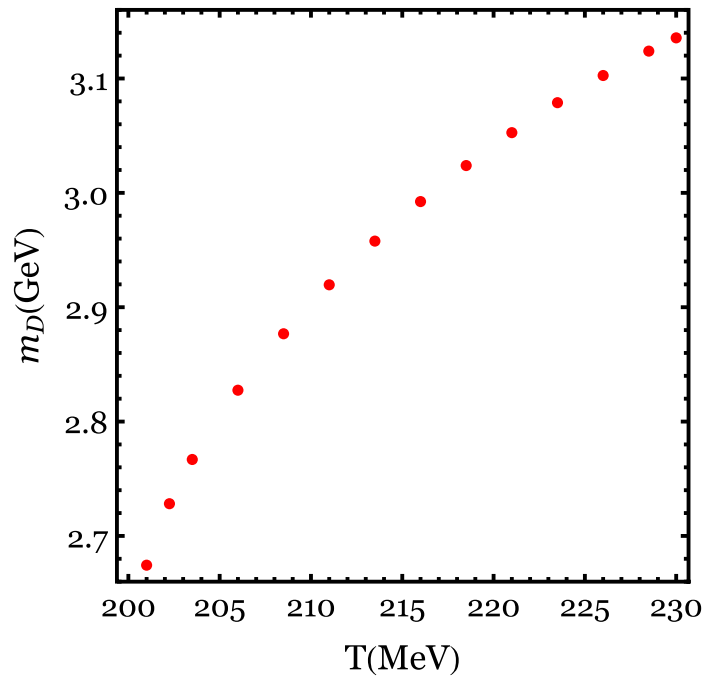

Fig. 6 Debye mass in $\mathrm{GeV}$ as a function of the temperature in $\mathrm{MeV}$

critical temperature $T_{c}$. One notes that for increasing temperature, the plasma becomes more and more screened since $m_{\mathrm{D}}$ is monotonically increasing. The model provides the values of the screening mass in the range $201 \mathrm{MeV} \leq T \leq 230 \mathrm{MeV}$, making it possible to investigate the region near the critical temperature.

An important support for the validity of the model comes from the check, in the previous section, of the $T=0$ limit. The value obtained for the glueball mass is consistent with lattice data. Other studies of screening in a nonAbelian plasma using holography can be found, for example, in $[31,32,40-42]$.

Acknowledgements N. B. is partially supported by $\mathrm{CNPq}$ (Brazil) and L. F. is supported by CAPES (Brazil).

Open Access This article is distributed under the terms of the Creative Commons Attribution 4.0 International License (http://creativecomm ons.org/licenses/by/4.0/), which permits unrestricted use, distribution, and reproduction in any medium, provided you give appropriate credit to the original author(s) and the source, provide a link to the Creative Commons license, and indicate if changes were made. Funded by SCOAP ${ }^{3}$.

\section{References}

1. J.M. Maldacena, Adv. Theor. Math. Phys. 2, 231 (1998)

2. J.M. Maldacena, Int. J. Theor. Phys. 38, 1113 (1999). arXiv:hep-th/9711200

3. S.S. Gubser, I.R. Klebanov, A.M. Polyakov, Phys. Lett. B 428, 105 (1998). arXiv:hep-th/9802109

4. E. Witten, Adv. Theor. Math. Phys. 2, 253 (1998). arXiv:hep-th/9802150

5. J. Polchinski, M.J. Strassler, Phys. Rev. Lett. 88, 031601 (2002). arXiv:hep-th/0109174

6. H. Boschi-Filho, N.R.F. Braga, Eur. Phys. J. C 32, 529 (2004). arXiv:hep-th/0209080
7. H. Boschi-Filho, N.R.F. Braga, JHEP 0305, 009 (2003). arXiv:hep-th/0212207

8. A. Karch, E. Katz, D.T. Son, M.A. Stephanov, Phys. Rev. D 74, 015005 (2006). doi:10.1103/PhysRevD.74.015005. arXiv:hep-ph/0602229

9. S.J. Brodsky, G.F. de Teramond, H.G. Dosch, J. Erlich, Phys. Rept. 584, 1 (2015). doi:10.1016/j.physrep.2015.05.001. arXiv:1407.8131 [hep-ph]

10. E. Witten, Adv. Theor. Math. Phys. 2, 505 (1998). arXiv:hep-th/9803131

11. H. Boschi-Filho, N.R.F. Braga, C.N. Ferreira, Phys. Rev. D 74, 086001 (2006). doi:10.1103/PhysRevD.74.086001. arXiv:hep-th/0607038

12. C.P. Herzog, Phys. Rev. Lett. 98, 091601 (2007). doi:10.1103/ PhysRevLett.98.091601. arXiv:hep-th/0608151

13. C.A. Ballon Bayona, H. Boschi-Filho, N.R.F. Braga, L.A. Pando Zayas, Phys. Rev. D 77, 046002 (2008). doi:10.1103/PhysRevD. 77.046002. arXiv:0705.1529 [hep-th]

14. U. Gursoy, E. Kiritsis, JHEP 0802, 032 (2008). doi:10.1088/ 1126-6708/2008/02/032. arXiv:0707.1324 [hep-th]

15. U. Gursoy, E. Kiritsis, F. Nitti, JHEP 0802, 019 (2008). doi:10. 1088/1126-6708/2008/02/019. arXiv:0707.1349 [hep-th]

16. U. Gursoy, E. Kiritsis, L. Mazzanti, F. Nitti, Nucl. Phys. B 820, 148 (2009). doi:10.1016/j.nuclphysb.2009.05.017. arXiv:0903.2859 [hep-th]

17. U. Gursoy, E. Kiritsis, L. Mazzanti, F. Nitti, Phys. Rev. Lett. 101, 181601 (2008). doi:10.1103/PhysRevLett.101.181601. arXiv:0804.0899 [hep-th]

18. U. Gursoy, E. Kiritsis, L. Mazzanti, F. Nitti, JHEP 0905, 033 (2009) doi:10.1088/1126-6708/2009/05/033. arXiv:0812.0792 [hep-th]

19. S.S. Gubser, A. Nellore, Phys. Rev. D 78, 086007 (2008). doi:10 1103/PhysRevD.78.086007. arXiv:0804.0434 [hep-th]

20. S.S. Gubser, A. Nellore, S.S. Pufu, F.D. Rocha, Phys. Rev. Lett. 101, 131601 (2008). doi:10.1103/PhysRevLett.101.131601. arXiv:0804.1950 [hep-th]

21. J. Alanen, K. Kajantie, V. Suur-Uski, Phys. Rev. D 80, 075017 (2009). doi:10.1103/PhysRevD.80.075017. arXiv:0905.2032 [hep-ph]

22. J. Alanen, K. Kajantie, V. Suur-Uski, Phys. Rev. D 80, 126008 (2009). doi:10.1103/PhysRevD.80.126008. arXiv:0911.2114 [hep-ph]

23. J. Alanen, K. Kajantie, Phys. Rev. D 81, 046003 (2010). doi:10. 1103/PhysRevD.81.046003. arXiv:0912.4128 [hep-ph]

24. J. Alanen, K. Kajantie, K. Tuominen, Phys. Rev. D 82, 055024 (2010). doi:10.1103/PhysRevD.82.055024. arXiv:1003.5499 [hep-ph]

25. K. Kajantie, M. Krssak, M. Vepsalainen, A. Vuorinen, Phys. Rev. D 84, 086004 (2011). doi:10.1103/PhysRevD.84.086004. arXiv: 1104.5352 [hep-ph]

26. J. Alanen, T. Alho, K. Kajantie, K. Tuominen, Phys. Rev. D 84, 086007 (2011). doi:10.1103/PhysRevD.84.086007. arXiv:1107.3362 [hep-th]

27. K. Kajantie, M. Krssak, A. Vuorinen, JHEP 1305, 140 (2013). doi:10.1007/JHEP05(2013)140. arXiv:1302.1432 [hep-ph]

28. D. Li, S. He, M. Huang, Q.S. Yan, JHEP 1109, 041 (2011). doi:10. 1007/JHEP09(2011)041. arXiv:1103.5389 [hep-th]

29. G. Boyd, J. Engels, F. Karsch, E. Laermann, C. Legeland, M. Lutgemeier, B. Petersson, Nucl. Phys. B 469, 419 (1996). doi:10.1016/ 0550-3213(96)00170-8. arXiv:hep-lat/9602007

30. P.B. Arnold, L.G. Yaffe, Phys. Rev. D 52, 7208 (1995). doi:10. 1103/PhysRevD.52.7208. arXiv:hep-ph/9508280

31. D. Bak, A. Karch, L.G. Yaffe, JHEP 0708, 049 (2007). doi:10. 1088/1126-6708/2007/08/049. arXiv:0705.0994 [hep-th]

32. S.I. Finazzo, J. Noronha, Phys. Rev. D 90(11), 115028 (2014). doi:10.1103/PhysRevD.90.115028. arXiv:1411.4330 [hep-th] 
33. S.I. Finazzo, Understanding strongly coupled non-Abelian plasmas using the gauge/gravity duality, Ph.D. Thesis, Universidade de São Paulo (2015). doi:10.11606/T.43.2015.tde-07042015-144444

34. N. Iqbal, H. Liu, Phys. Rev. D 79, 025023 (2009). doi:10.1103/ PhysRevD.79.025023. arXiv:0809.3808 [hep-th]

35. U. Grsoy, I. Iatrakis, E. Kiritsis, F. Nitti, A. O'Bannon, JHEP 1302, 119 (2013). doi:10.1007/JHEP02(2013)119. arXiv:1212.3894 [hep-th]

36. H.R. Grigoryan, P.M. Hohler, M.A. Stephanov, Phys. Rev. D 82, 026005 (2010). doi:10.1103/PhysRevD.82.026005. arXiv:1003.1138 [hep-ph]

37. N.R.F. Braga, M.A. Martin Contreras, S. Diles, Eur. Phys. J. C 76(11), 598 (2016). doi:10.1140/epjc/s10052-016-4447-4. arXiv:1604.08296 [hep-ph]
38. C.J. Morningstar, M.J. Peardon, Phys. Rev. D 60, 034509 (1999). doi:10.1103/PhysRevD.60.034509. arXiv:hep-lat/9901004

39. Y. Chen et al., Phys. Rev. D 73, 014516 (2006). doi:10.1103/ PhysRevD.73.014516. arXiv:hep-lat/0510074

40. C. Hoyos, S. Paik, L.G. Yaffe, JHEP 1110, 062 (2011). doi:10. 1007/JHEP10(2011)062. arXiv:1108.2053 [hep-th]

41. A. Singh, A. Sinha, Nucl. Phys. B 864, 167 (2012). doi:10.1016/j. nuclphysb.2012.06.013. arXiv:1204.1817 [hep-th]

42. O. Andreev, Phys. Rev. D 94(12), 126003 (2016). doi:10.1103/ PhysRevD.94.126003. arXiv:1608.08026 [hep-ph] 